\title{
Percepção do Residente em Relação a Turismo e Megaevento: análise bibliométrica de periódicos internacionais e latino-americanos
}

\author{
Resident Perception Toward Tourism and Mega Event: bibliometric analysis of \\ international and Latin American journals \\ Percepción del Residente Hacia el Turismo y Mega Evento: análisis bibliométrica de los \\ periódicos internacionales y de América Latina
}

\author{
Marcelo Chiarelli Milito ${ }^{1}$ \\ Sérgio Marques ${ }^{2}$ \\ Mauro Lemuel Alexandre ${ }^{3}$
}

\begin{abstract}
Resumo
Entender a percepção da comunidade residente sobre o turismo, se mostra relevante por ser um grupo que é impactado e impacta esse nicho de mercado, com ênfase em regiões que possuem uma estrutura social instável e que estão investindo em turismo e megaeventos, como o continente latino-americano. Para entender o desenvolvimento do conhecimento de determinado fenômeno, se recorre a investigações sobre a comunicação científica que versam sobre os avanços na área de interesse. Assim, a presente pesquisa objetiva caracterizar os contrastes nos estudos científicos internacionais e latino-americanos, que versam sobre a percepção dos residentes frente ao turismo e megaeventos. A metodologia utilizada é de análise bibliométrica na comparação da produção dos principais periódicos das duas regiões supracitadas, nos últimos dez anos, através de uma perspectiva quantitativa e qualitativa, de característica descritiva. Revela-se que as pesquisas sobre o turismo através do prisma da comunidade local, estão relevantes em todos os periódicos abordados, mas com diferenças acentuadas quanto às metodologias de pesquisa usadas nas revistas do continente latino e as globais. Conclui com a proposição da aplicação do marco teórico internacional em âmbito continental, principalmente no que tange a realização dos megaeventos.
\end{abstract}

Palavras-chave: Turismo. Megaeventos. Residentes. América Latina. Bibliometria.

\footnotetext{
${ }^{1}$ Bacharel em Turismo pela Universidade Federal do Rio Grande do Norte; Master em Dirección y Planificación del Turismo por la Universitat Rovira i Virgili; Mestrando em Turismo da Universidade Federal do Rio Grande do Norte, Bolsista CAPES. Brasil. E-mail

${ }^{2}$ Doutor em Agronomia pela Universidade Estadual Paulista Júlio de Mesquita Filho. Professor adjunto da Universidade Federal do Rio Grande do Norte; Professor do Programa de Pós-Graduação em Turismo da Universidade Federal do Rio Grande do Norte. Brasil. E-mail

${ }^{3}$ Doutor em Ciências em Engenharia de Produção pela Universidade Federal do Rio de Janeiro. Professor Associado da Universidade Federal do Rio Grande do Norte. Professor do Programa de Pós-Graduação em Turismo da Universidade Federal do Rio Grande do Norte. Brasil. E-mail
} 


\begin{abstract}
It is relevant to understand the perception of resident communities towards tourism given that these groups both impact and are impacted by this niche market, especially in regions with unstable social structure and which are investing in tourism and mega events, such as Latin American countries. To better understand the state-of-knowledge on this phenomenon, we investigate the scientific literature for evidence of advances in this area. The objective of this study is to confront international scientific studies and those in Latin American relating to the perception of residents towards tourism and Mega Events. To this end the methodology used consisted of a comparative bibliometric analysis of output from the primary journals in the two aforementioned regions within the last ten years, by describing quantitative and qualitative elements. Reveal that tourism research as viewed through the prism of local communities is relevant to all the journals examined, however there are marked differences in research methodologies among the Latin American journals compared and the international journals. Concludes with the proposition of a international theoretical application in a continental scope, mainly in the implementation of mega events.
\end{abstract}

Keywords: Tourism. Mega Events. Residents. Latin America. Bibliometry.

\title{
Resumen
}

Entender la percepción de la comunidad residente en el turismo es relevante por apuntar en un grupo que impacta y es impactado a este nicho de mercado, centrándose en las regiones que tienen una estructura social inestable y tiene expresiva inversión en turismo y mega eventos, como el continente de América Latina. Para entender el desarrollo del conocimiento de un fenómeno, es necesario investigar la comunicación de la ciencia que tienen que ver con los avances en el área de interés. Así, este estudio objetiva confrontar a los estudios internacionales y de América Latina que versan en la percepción del residente hacia el turismo y mega eventos. Se utilizó la metodología de análisis bibliométrica para comparar la producción de las principales revistas de las dos regiones mencionadas, en los últimos diez años, a través de un punto de vista cualitativo y cuantitativo, con característica descriptiva. La investigación revela que la análisis del turismo a través del prisma de la comunidad local son importantes en todas las revistas incluidas, pero con marcadas diferencias en las metodologías utilizadas en las revistas de investigación en el continente de América y en el mundo. Se concluye con la proposición de una aplicación teórica internacional para un alcance continental, principalmente en cuanto a la implementación de mega eventos.

Palabras-clave: Turismo. Mega eventos. Residentes. América Latina. Bibliometria.

\section{Introdução}

O desenvolvimento de uma ideia normatiza-se no momento em que a comunidade científica toma conhecimento desses dados. Esses são publicados por meio de uma comunicação científica, difusão essa que tomou a vanguarda dos conhecimentos na atualidade. Dentre as 
formas de comunicação científica, os periódicos tomam um caráter principal dos resultados das pesquisas, principalmente os mais bem conceituados, pois se mostram como o marco teórico dos diversos campos do conhecimento (SILVA, 2005).

Para Hall (2011) juntamente com a informetria, o estudo dos aspectos quantitativos da informação, a bibliometria, o estudo quantitativo das publicações, tornou-se uma questão cada vez mais significativa em estudos de turismo. Autores como Rejowski (2011) destacam a importância dos mapeamentos das pesquisas no setor, que possibilitam identificar pontos fortes e fracos em determinado campo de estudo e perspectivas sobre o progresso científico e formas de aprimorá-lo.

Segundo Estivals (1985) as medidas se referem aos objetivos, aos fenômenos ou fatos, as relações ou leis, assim, o que deve ser estimado em uma pesquisa bibliométrica é a "flutuação do interesse coletivo sobre a matéria (p.37)". Sendo assim, o objetivo desse tipo de pesquisa define as categorias de análise, pois resultará no foco das flutuações de interesse a serem mensuradas.

Entender o posicionamento da comunidade local está em pauta tanto nos campos teóricos como empíricos, dentro e fora do campo do turismo. Dessa forma, o presente estudo vem no intuito de mapear a flutuação dos fluxos do interesse científico sobre a percepção dos residentes frente ao turismo e megaeventos, bem como identificar lacunas a serem preenchidas dentro dessa tipologia de pesquisa no continente latino-americano.

Para tal, objetivou-se caracterizar os contrastes nos estudos científicos internacionais e latinoamericanos que versam sobre a percepção dos residentes frente ao turismo e megaeventos. As categorias de análise abordadas para constituir o objetivo central do estudo são: a) Quantificar as pesquisas sobre o tema b) Identificar as metodologias usadas c) Identificar autores e regiões das pesquisas d) Apontar sugestões de caminhos nessa tipologia de pesquisa para o continente em foco.

O escopo da presente pesquisa está composto por um contexto inicial em que se interrelaciona o turismotos, megaeventos, residentes e o continente latino-americano, seguido das especificações metodológicas que validaram tal estudo, a apresentação dos resultados, e por fim, a conclusão. 


\section{Turismo e Megaeventos: concepção e contexto}

O turismo tem na raiz de sua atividade o movimento, onde as pessoas, ao se locomoverem fora do eixo habitual, geram rendas vinculadas a esses deslocamentos e até investimentos prévios em infraestrutura para acomodar essa possível demanda, principalmente no caso dos megaeventos. Proporcional a esse tipo de ação, forma-se uma reação por parte dos locais influenciados nessa dinâmica, positiva ou negativa, a depender do contexto que esse fenômeno se apresenta. Assim, entender essas reações vem como um fator chave para o sucesso de projetos no setor.

O turismo é um dos fenômenos em discussão na atualidade, tanto nas arenas teóricas como empíricas, por seu recente progresso mercantil, passando nas últimas décadas mercados consolidados como o petróleo e o automobilístico, além de se tornar o maior gerador de empregos diretos e indiretos da atualidade (Organização Mundial do Turismo, 2011).

Dessa maneira, o investimento no potencial turístico de uma localidade é um posicionamento estratégico constantemente adotado hoje na economia. Uma das formas de atrair a demanda turística internacional e desenvolver economicamente a região são os megaeventos, pois transformam a infraestrutura local em prol do evento e propaga uma imagem turística em âmbito global (BRASIL, 2011).

Essa tipologia de atividade, por sua natureza múltipla e de alta complexidade, ainda está com seu conhecimento aquém da normatização dos efeitos, dando margem a constantes estudos acadêmicos para um entendimento da área (PANOSSO, 2005).

Uma dos campos do turismo que aponta peculiaridades, é o fato da hospitalidade do residente ser um dos fatores decisivos para a imagem turística de um destino, (ALEGRE, J. e GARAU, J., 2010; MARREIRO, C. M. e MARQUES Jr, S. 2011). Congruente com as pesquisas que identificam os residentes como um dos stakeholders primários e que deve ter seus interesses contemplados em um projeto turístico, a fim de evitar conflitos que arriscam o sucesso da empreitada (CURRIE, R. R., SEATON, S. e WESLEY, F., 2009; DREDGE, D., 2010). 
Apesar do entendimento que a comunidade impacta de maneira relevante esse tipo de mercado, os impactos sofridos pela comunidade local ainda estão em processo de discussão. Autores como Krippendorf (2003) apontam o turismo como transformador de localidades bucólicas em objetos de consumo desenfreado, que alimentam a antipatia dos autóctones por ver seu habitat transformado de maneira contrária ao seu gosto, além da impressão de estarem sendo invadidos. Ao mesmo tempo autores como Palomo (2000) indicam que o turismo deveria ser uma atividade prioritária no século XXI, pois tem um potencial de crescimento que não circunde apenas as nações desenvolvidas, possibilitando uma maior equidade socioeconômica global.

Apesar da divergência entre os autores quanto às formas de impacto do turismo sobre os residentes e como essa inter-relação de impacto comporta-se, o campo empírico continua em franco crescimento, principalmente nos países em desenvolvimento. Como referência, uma das fontes financeiras para o desenvolvimento do continente latino americano, o Banco Interamericano de Desenvolvimento (BID), aprovou nos últimos dez anos (2002 até 2011) o financiamento de 140 projetos no setor de turismo, com destaque para projetos como o “PRODETUR nordeste II - Brasil” no valor de 400 milhões de dólares (BID, 2012) 4 .

Em convergência com essa busca do turismo em localidades subdesenvolvidas, a OMT (2010) aponta que as chegadas internacionais de turistas no ano de 2009 foram representadas em 46,9\% nas economias emergentes, um progresso vultoso se compararmos os dados de 1970, em que havia apenas $15 \%$ das chegadas internacionais para as nações subdesenvolvidas. Como exemplo cabe expor a tabela 1 com os dados do turismo na América do sul e a crescente participação desse mercado mundial a partir do ano de 2002:

\footnotetext{
${ }^{4}$ Dados extraídos da ferramenta de busca disponível no site oficial do BID: http://www.iadb.org/pt/projetos/busca-avancada,1301.html?adv=true
} 
Tabela 1 - Chegada de turistas internacionais, Mundo e América do Sul.

\begin{tabular}{|c|c|c|c|c|}
\hline \multirow{3}{*}{ Ano } & \multicolumn{4}{|c|}{ Turistas (milhöes de chegadas) } \\
\hline & \multicolumn{2}{|c|}{ Mundo } & \multicolumn{2}{|c|}{ América do Sul } \\
\hline & Total & Variação anual (\%) & Total & $(\%)$ \\
\hline 1998 & & - & & - \\
\hline 1999 & & 3,77 & & $(2,58)$ \\
\hline 2000 & & 6,00 & & 0,66 \\
\hline 2001 & & $(0,10)$ & & $(3,95)$ \\
\hline 2002 & & 2,96 & & $(13,01)$ \\
\hline 2003 & & $(1,74)$ & & 7,87 \\
\hline 2004 & & 9,89 & & 18,40 \\
\hline 2005 & & 5,03 & & 12,20 \\
\hline 2006 & & 5,39 & & 2,75 \\
\hline 2007 & & 6,73 & & 6,95 \\
\hline 2008 & & 1,93 & & 4,00 \\
\hline
\end{tabular}

Fonte: Brasil 2010, p. 5.

Assim como o turismo, o continente latino-americano também possui peculiaridades que devem ser observadas, principalmente no que tange os aspectos sociais. Autores como Galeano (1982) discorrem sobre a temática da formação histórica dos países latino americanos, que tiverem uma colonização ibérica extrativista, onde originou as formas que essa estrutura reverberou nos distintos processos de poder, que vai da imposição do colonizador pela institucionalização do monopólio e estímulo a rivalidade interna entre os países latinos, até as constantes tentativas de insurreição social.

No campo empírico cabe salientar o índice de homicídios intencionais (por 100.000 habitantes) apresentados pelo World Economic Bank (WEB, 2012), que no último senso oficial e global sobre esse tópico, ano de 2008, apontou uma média mundial de 7 (sete) homicídios. Na América Latina esse índice se apresentou com 25 (vinte e cinco), ou seja, o continente em estudo possui mais que o triplo de homicídios que a média global. No caso do Brasil, país inserido no continente em estudo, atual sexta maior economia do mundo (Centre for Economics and Business Research, 2012) e que irá receber dois dos maiores megaeventos da atualidade, a Copa do Mundo FIFA em 2014 e Jogos Olímpicos em 2016, observa-se um índice de homicídios de 30 (trinta), dados esses relevantes no presente contexto ao comparar 
com os países da União Europeia, principais clientes desses eventos, que possuem uma média de homicídios em torno de 1 (um), ou seja, serão recebidos em uma região onde se mata 30 vezes mais que seu país de origem (WEB, 2012) ${ }^{5}$.

Fatos como esse expõe o problema social que ainda persiste na região e abre margem para explicação como a de Brandão (2004), que responsabiliza o estruturalismo latino-americano como causador de uma malformação no arcabouço desse continente, com grande capacidade de persistência. Com isso, expõe-se uma lacuna sobre o entendimento da reação dessa sociedade ao receber o turismo e megaeventos, pois a história sociopolítica latino-americana está repleta de confrontos sociais e que podem refletir em um risco para os investimentos turísticos que necessitam de uma imagem positiva, que em grande parte, será emitida pela hospitalidade local.

Ao considerar que os artigos publicados em periódicos científicos é a forma padrão de disseminação de resultados aceitáveis nas diversas áreas do conhecimento, e que atualmente, inclusive a produção técnica e artística é encontrada no formato de artigos científicos (Silva, 2005), surge a oportunidade de analisar como esse meio de comunicação trata o referido tema, em um viés que pretende caracterizar os caminhos tomados pelos pesquisadores para exploração da problemática exposta.

\section{Metodologia de Análise}

Os estudos que pretendem quantificar os processos de comunicação escrita é uma forma de análise bibliométrica, uma tipologia de estudo pertinente quando o intuito é medir de forma coordenada os conhecimentos de determinado aspecto (OTLET, 1986). Essa tipologia de estudo está sendo usada no campo do turismo por autores relevantes em âmbito internacional como Hall (2011) e nacional como Rejowski (2010), pois fornece uma visão do crescimento de uma determinada área de estudo, além de identificar as formas e regiões que esses

\footnotetext{
${ }^{5}$ Dados extraídos da ferramenta de busca disponível no site oficial do WEB: http://data.worldbank.org/
} 
conhecimentos são produzidos. Perspectiva essa convergente com um nicho em franco crescimento empírico e teórico como o turismo.

Como o presente trabalho possui, como objetivo central, caracterizar os contrastes da comunicação científica entre duas regiões e, ao considerar que as pesquisas descritivas têm como principal objetivo descrever as características de determinada população ou fenômeno ou estabelecimento de relações entre variáveis (GIL, 1994), pode-se considerar o presente estudo de caráter descritivo. Para analisar o objeto de estudo, foi feita uma análise de conteúdo, seguida de uma tabulação dos dados obtidos em tabelas e gráficos, favorecendo uma abordagem qualitativa e quantitativa.

A investigação focou nos periódicos científicos, pois apesar de existirem vários meios de comunicação científica que podem ser analisados nessa tipologia de pesquisa, os mais usados no campo do turismo são os periódicos (HALL, 2011). Foi selecionado como universo da pesquisa, as revistas mais relevantes editadas nas regiões em estudo, fator que possibilita uma visão comparativa do marco teórico abordado em cada localidade.

Para tal discriminação foi considerada a instituição de Coordenação de Aperfeiçoamento Pessoal de Nível Superior (CAPES), em que foi selecionada apenas as revistas de turismo, editadas no continente e que possuem avaliação acima do conceito B3. Para as revistas internacionais, foi levado em conta a pesquisa de Ryan (2005) que faz um ranking das revistas mais relevantes de turismo em âmbito mundial. Para viabilizar a pesquisa, foi feito mais um recorte, espacial e temporal, a disponibilidade on-line desses periódicos nos últimos dez anos, período que se apresenta uma crescente participação do mercado turístico no continente em estudo, conforme tabela 1. A partir dos critérios supracitados a abrangência da pesquisa se apresenta na tabela a seguir: 
Tabela 2: Recorte da pesquisa

\begin{tabular}{c|c|c|c|c}
\hline Âmbito & Revista & Ranking & Disponibilidade no período & Total de artigos no período \\
\hline Mundial & Annals of Tourism Research & $1^{\mathrm{a}}$ & $2002-2011$ & 503 \\
\cline { 2 - 5 } & Tourism Manegement & $2^{\mathrm{a}}$ & $2001-2011$ & 841 \\
\hline $\begin{array}{c}\text { América } \\
\text { Latina }\end{array}$ & Estudios y Perspectivas en Turismo & B1 & $2003-2011$ & 240 \\
\cline { 2 - 5 } & Turismo em Análise & B2 & $2008-2011$ & 117 \\
\cline { 2 - 5 } & Turismo Visão e Ação & B3 & $2002-2011$ & 201 \\
\hline
\end{tabular}

Fonte: Dados da pesquisa através de Ryan (2005), CAPES (2011) e sites das respectivas revistas.

A coleta de dados das revistas internacionais foi feita através da ferramenta de busca científica "Science Direct", as revistas do continente latino-americano foram retiradas diretamente dos sites de cada revista, ambos extraídos durante o período de 01/11/2011 até 30/04/2012. Os dados buscados foram referenciados pelos objetivos do estudo, que teve como foco central a abordagem "a percepção do residente sobre o turismo" nas revistas selecionadas, o que possibilitou a quantificação do estudo, caracterização das metodologias usadas e identificação dos países e autores dos artigos, além de uma proposta de pesquisa para o continente.

Devido á complexidade de padronização semântica dos termos, seja no título, resumo ou palavras-chaves, para caracterizar trabalhos que versam sobre a temática proposta, se fez necessário uma análise individual de todos os artigos. Conforme aponta a tabela 2, foi analisado um total de 1344 artigos nas revistas internacionais e 558 artigos nas revistas do continente, em dois momentos distintos, o primeiro de identificação dos artigos e segundo com a categorização dos documentos identificados.

No primeiro momento, esse montante foi filtrado em três etapas: análise do título, seguido de uma análise do resumo e, por fim, uma análise do corpo do texto. Somente os documentos que passaram do filtro antecessor seguiram na triagem, em caso de dúvida, o artigo seguia nas etapas de seleção. Vale ressaltar que, o critério para identificação do conteúdo, em cada uma das etapas, foram pesquisas focadas na "percepção (posicionamento, atitudes ou afins) dos 
residentes", artigos que abordavam os autóctones sem ter como pesquisa central o ponto de vista dos mesmos, não foram consideradas dentro da classificação.

Ao final, identificou-se um total de 52 artigos internacionais e 15 continentais sobre o tema, que num segundo momento, foram submetidos para identificação das categorias de análise da presente pesquisa. Todos os dados foram copilados e trabalhados através da ferramenta digital Microsoft Excel.

\section{Abordagem em Periódicos sobre Turismo e Residentes}

Os resultados estão apresentados conforme a ordem das categorias de análise propostas: quantificar os artigos no tema em relação ao universo total da pesquisa, seguido de uma abordagem sobre as metodologias de pesquisa identificadas nos artigos sobre o tema, acompanhada pela identificação dos autores e regiões das investigações e, por último, a partir dos dados levantados, sugestões de pesquisa para o continente latino-americano. Nessas diversas etapas foi seguido o propósito geral da pesquisa, contrastar os estudos publicados entre as duas regiões analisadas.

Mensurar a quantidade e a frequência que as revistas científicas relevantes, conforme exposto na tabela 2, vêm publicando artigos sobre a visão do residente sobre o turismo, abre a possibilidade do entendimento da saliência do referido tema, proposta essa que resultou na tabela 3:

Tabela 3: Quantidade e frequência nas revistas latinas.

\begin{tabular}{|c|c|c|c|c|c|c|c|c|c|c|c|c|c|c|c|c|c|c|c|c|}
\hline & \multicolumn{2}{|c|}{2002} & \multicolumn{2}{|c|}{2003} & \multicolumn{2}{|c|}{2004} & \multicolumn{2}{|c|}{2005} & \multicolumn{2}{|c|}{2006} & \multicolumn{2}{|c|}{2007} & \multicolumn{2}{|c|}{2008} & \multicolumn{2}{|c|}{2009} & \multicolumn{2}{|c|}{2010} & \multicolumn{2}{|c|}{2011} \\
\hline & $\mathbf{Q}$ & $\%$ & $\mathbf{Q}$ & $\%$ & $\mathbf{Q}$ & $\%$ & $\mathbf{Q}$ & $\%$ & $\mathbf{Q}$ & $\%$ & $\mathbf{Q}$ & $\%$ & $\mathbf{Q}$ & $\%$ & $\mathbf{Q}$ & $\%$ & $\mathbf{Q}$ & $\%$ & $\mathbf{Q}$ & $\%$ \\
\hline I & - & - & 0 & 0 & 0 & 0 & 0 & 0 & 0 & 0 & 0 & 0 & 0 & O & 2 & 5,7 & 3 & 6,1 & 1 & 1,5 \\
\hline II & - & & - & - & - & - & - & - & - & - & - & - & 2 & 7,4 & 0 & 0 & 1 & 3,3 & 0 & 0 \\
\hline III & 1 & 4,8 & 1 & 6,3 & 0 & 0 & 0 & 0 & 1 & 4,8 & 1 & 4,5 & 1 & 4,8 & 1 & 4,8 & 0 & 0 & 0 & 0 \\
\hline $\mathbf{T}$ & 1 & 4,8 & 1 & 3,1 & 0 & 0 & 0 & 0 & 1 & 2,7 & 1 & 2,6 & 3 & 5,0 & 3 & 3,5 & 4 & $\begin{array}{l}, 0 \\
, 0\end{array}$ & 1 & 0,8 \\
\hline
\end{tabular}

Legendas: I - Estudios y perspectivas en Turismo/ II- Turismo em Análise /

III- Turismo Visão e Ação/ T - total / Q- Quantidade / \% - Frequência por cento.

Fonte: Dados da pesquisa, 2012. 
Identifica-se na tabela 3 um total de 15 artigos sobre o tema nos últimos dez anos nas principais revistas de turismo do referido continente, o que significa uma média de 1,5 estudos publicados por ano. Em porcentagem, uma média de $2,7 \%$, de forma crescente e contínua a partir do ano de 2006, dando ideia de um foco que está presente e em progresso.

Ao seguir o mesmo raciocínio, a fim de estabelecer um comparativo com as revistas mais relevantes de âmbito mundial, conforme tabela 2, expõe-se a tabela 4:

Tabela 4: Quantidade e frequência nas revistas internacionais.

\begin{tabular}{|c|c|c|c|c|c|c|c|c|c|c|c|c|c|c|c|c|c|c|c|c|}
\hline & \multicolumn{2}{|c|}{2002} & \multicolumn{2}{|c|}{2003} & \multicolumn{2}{|c|}{2004} & \multicolumn{2}{|c|}{2005} & \multicolumn{2}{|c|}{2006} & \multicolumn{2}{|c|}{2007} & \multicolumn{2}{|c|}{2008} & \multicolumn{2}{|c|}{2009} & \multicolumn{2}{|c|}{2010} & \multicolumn{2}{|c|}{2011} \\
\hline & $\mathbf{Q}$ & $\%$ & $\mathbf{Q}$ & $\%$ & $\mathbf{Q}$ & $\%$ & $\mathbf{Q}$ & $\%$ & $\mathbf{Q}$ & $\%$ & $\mathbf{Q}$ & $\%$ & $\mathbf{Q}$ & $\%$ & $\mathbf{Q}$ & $\%$ & $\mathbf{Q}$ & $\%$ & $\mathbf{Q}$ & $\%$ \\
\hline I & 4 & 7,4 & 2 & 4,3 & 1 & 1,8 & 5 & 9,8 & 2 & 3,9 & 2 & 4,0 & 1 & 2,1 & 1 & 3,4 & 0 & 0 & 4 & 6,0 \\
\hline II & 2 & 3,8 & 4 & 7,0 & 1 & 1,5 & 2 & 2,5 & 5 & 5,2 & 4 & 3,6 & 2 & 2,2 & 2 & 2,5 & 2 & 2,4 & 6 & 4,8 \\
\hline $\mathbf{T}$ & 6 & 5,7 & 6 & 5,8 & 2 & 1,7 & 7 & 5,4 & 7 & 4,7 & 6 & 3,8 & 3 & 2,2 & 3 & 2,7 & 2 & 1,5 & 10 & 5,2 \\
\hline
\end{tabular}

Legenda: I - Annals of tourism Research / II- Tourism Manegement / Q- Quantidade / $\%$ - Frequência por cento

Fonte: Dados da pesquisa, 2012.

A tabela 4 mostra um total de 52 artigos sobre o tema nos últimos dez anos nas revistas mais relevantes de turismo no mundo, que representa uma publicação média de 5,2 estudos por ano, em termos de porcentagem 3,9\% dos artigos nas revistas pesquisadas usavam como foco a percepção (posicionamento, atitudes ou afins) da comunidade receptora frente à atividade turística. Outro fator observado é a continuidade, todos os anos apontaram ao menos 2 artigos sobre o tema, sendo que o último ano de 2011, apresentou a maior quantidade bruta de artigos sobre o tema.

Através da observação de alguns trabalhos que antecedem os anos em estudo e em conjunto com a tabela 3 e 4, nota-se que o interesse pelo tema é anterior ao ano de 2002. De forma geral esse tipo de pesquisa está sendo abordada, mas não de maneira crescente ou decrescente, e sim constante, há mais tempo que o recorte contemplou, assim, essa é uma temática que permeia os estudos internacionais em turismo a mais de uma década. 
Em um enfoque comparativo, nas revistas internacionais foi identificado uma quantidade de artigos relacionados superior, 52 periódicos internacionais para 15 do continente, conforme tabela 4, mas o percentual apresenta menos contrastes devido à quantidade total de publicações serem proporcionalmente distintas. De maneira geral se encontrou uma superioridade de adoção na quantidade e, em menor contraste, percentual das revistas internacionais sobre o tema.

No que tange aos aspectos metodológicos usados nesses 67 artigos identificados, foram analisadas a natureza da pesquisa e a teoria base usada, relevantes para o entendimento das formas que essas pesquisas estão sendo conduzidas.

Quanto à natureza da pesquisa foram identificadas basicamente metodologias quantitativas, qualitativas e "quantitativas - qualitativas". Dados esses expostos na tabela 5:

Tabela 5: Comparativo quanto a natureza da pesquisa

\begin{tabular}{l|l|l|l|l|l|l}
\hline Tipologia & \multicolumn{2}{|l|}{ Quantitativas } & \multicolumn{2}{l|}{ Qualitativas } & \multicolumn{2}{l}{ Qualitativa e Quantitativa } \\
\hline Periódicos & Q & $\%$ & Q & $\%$ & Q & $\%$ \\
\hline Continente & 3 & 20,0 & 8 & 53,3 & 4 & 26,7 \\
\hline Internacional & 31 & 59,6 & 9 & 17,3 & 12 & 23,1 \\
\hline
\end{tabular}

Legenda: Q- Quantidade /\%- Porcentagem

Fonte: Dados da pesquisa, 2012.

A tabela 5 aponta um contraste expressivo quanto às formas de abordagem metodológicas para o referido tema, enquanto a maioria dos artigos latinos trabalha com técnicas qualitativas, a maioria dos artigos internacionais usam as técnicas quantitativas de análise.

No intuito de aprofundar o entendimento nas diferentes formas de pesquisa, foi verificada a teoria base que norteia os instrumentos das pesquisas no tema, foi identificada uma grande variedade de metodologias, com ênfase para a Social Exchange Theory (SET) e a Etnografia, conforme tabela 6: 
Tabela 6: Comparativo quanto à teoria do instrumento da pesquisa

\begin{tabular}{l|l|l|l|l|l|l}
\hline Tipologia & \multicolumn{2}{|l|}{ SET } & \multicolumn{2}{l|}{ Etnografia } & \multicolumn{2}{l}{ Outras } \\
\hline Periódicos & Q & $\%$ & Q & $\%$ & Q & $\%$ \\
\hline Continente & 1 & 6,7 & 3 & 20,0 & 11 & 73,3 \\
\hline Internacional & 27 & 51,9 & 7 & 13,4 & 18 & 34,7 \\
\hline
\end{tabular}

Legenda: Q- Quantidade /\%- Porcentagem

Fonte: Dados da pesquisa, 2012.

Nos periódicos da América latina não observou-se maioria absoluta em uma tipologia de teoria base para validar o instrumento de pesquisa, a que mais apareceu foi a Etnografia em $20 \%$ dos trabalhos. Nos periódicos internacionais houve uma maioria absoluta, o SET, usado em mais da metade dos estudos identificados sobre o tema, apresenta-se assim como teoria padrão para explicar o ponto de vista do residente frente ao turismo. Vale salientar que o SET é um instrumento primordialmente quantitativo e a Etnografia é um instrumento primordialmente qualitativo. Esse fator pode explicar o contraste marcante entre as metodologias quantitativas e qualitativas usadas nos dois contextos em estudo.

A relação dos países em que foram aplicados os estudos sobre o tema é oportuno, pois revela as localidades que estão sendo monitoradas. Essa perspectiva, a partir das revistas do continente, está no gráfico 1:

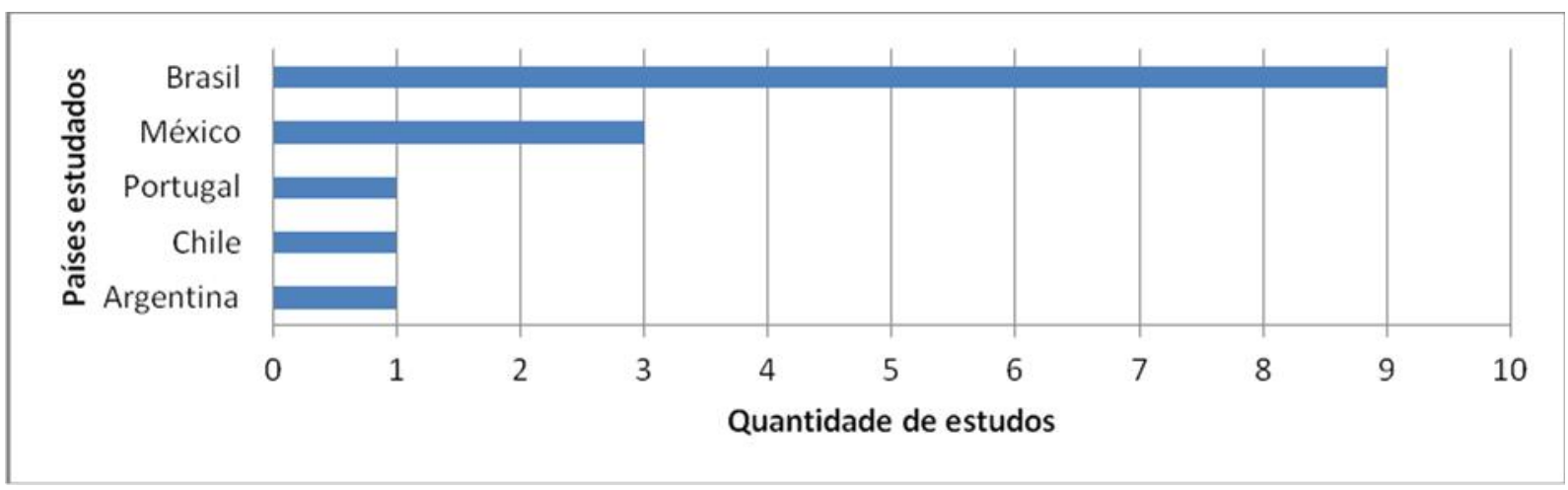

Gráfico 1: Países estudados pelas revistas do continente latino

Fonte: Dados da pesquisa, 2012. 
O Brasil apresenta a maior quantidade de trabalhos publicados sobre o tema nas revistas latinas, mas cabe ressaltar que duas das três revistas em análise são publicadas no Brasil. Outro fator que pode justificar é a barreira linguística, o único artigo não-brasileiro nas revistas brasileiras analisadas é um artigo de Portugal, ou seja, não foram identificados publicações de outros países latinos nas revistas brasileiras.

Da mesma forma foram analisados os países pesquisados nas revistas internacionais, também no intuito de entender quais regiões estão sendo as mais investigadas:

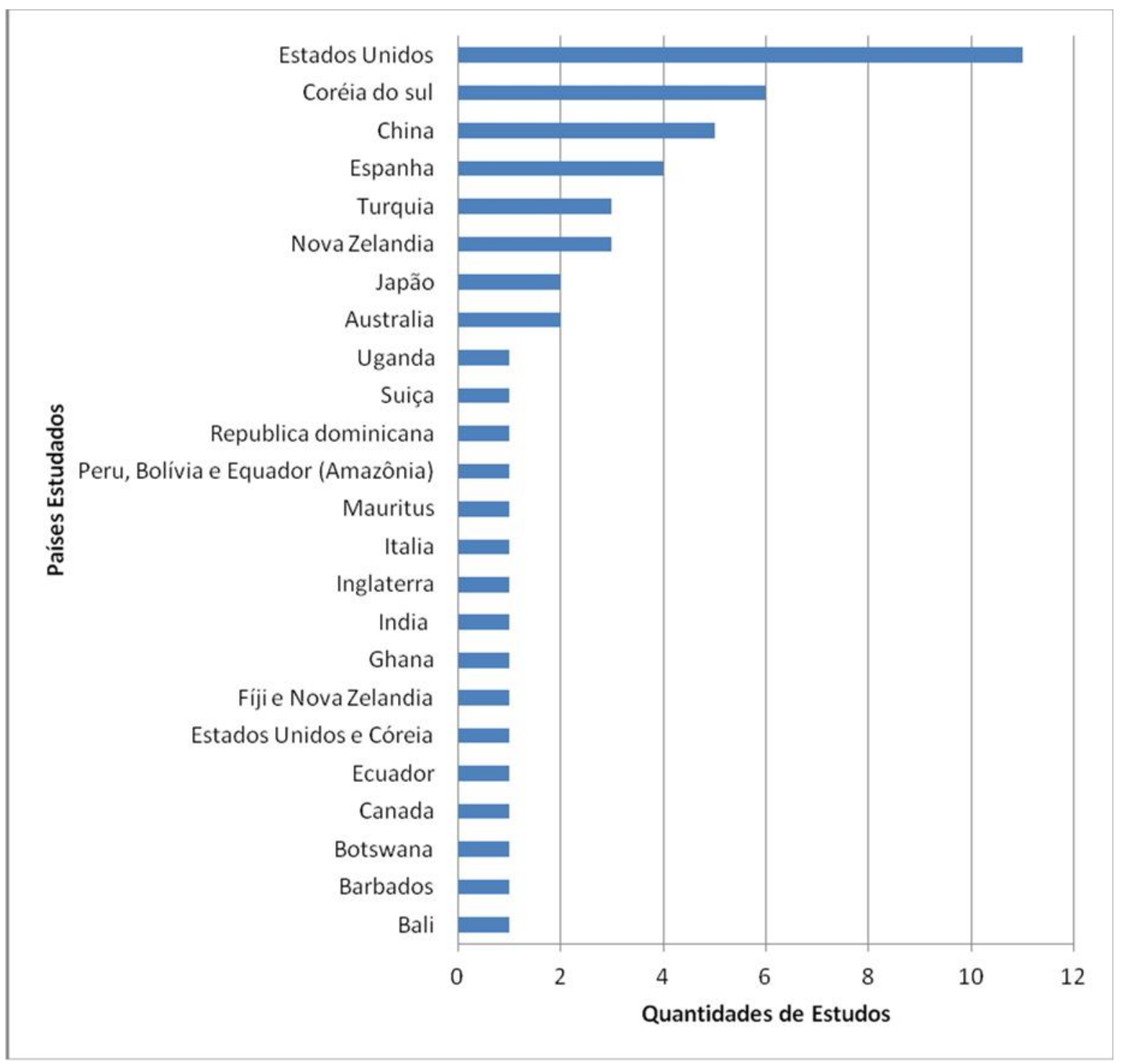

Gráfico 2: Países estudados pelas revistas internacionais

Fonte: Dados da pesquisa, 2012. 
É de se esperar que as revistas mais relevantes em âmbito mundial de determinada área, no presente caso do turismo, apresentem uma diversidade quanto às regiões estudadas, mas esse aspecto reforça a noção que o tema em estudo está sendo tratado nas mais representativas nações da atualidade, como Estados Unidos, Coreia do Sul e China, inclusive alguns estudos fazem uma comparação entre países para analisar as diferentes percepções da comunidade. Os continentes com menor participação é a América Latina e África, menos de $10 \%$ cada um.

Uma das flutuações de interesses comumente mensurados nas pesquisas bibliométricas é em relação aos autores. No presente levantamento, por buscar como determinado tema está sendo trabalhado, se enfatizou autores que repetidamente publicaram na última década sobre o assunto em questão, na intenção de identificar o quanto a temática está focada por um grupo constante de pesquisadores especialistas no assunto.

Nas revistas latinas, dos 37 autores publicados, o único que foi identificado com repetição foi Ontiveros, que publicou dois artigos, um sobre uma região turística no México e o segundo trabalho aplicou um estudo em um megaevento, que também ocorre no México, ambos os artigos na revista Estudios y Perspectivas em Turismo.

Nas revistas internacionais se observou 10 pesquisadores, no universo de 166, que publicaram mais de um artigo sobre o tema na última década, conforme exposto no gráfico abaixo: 


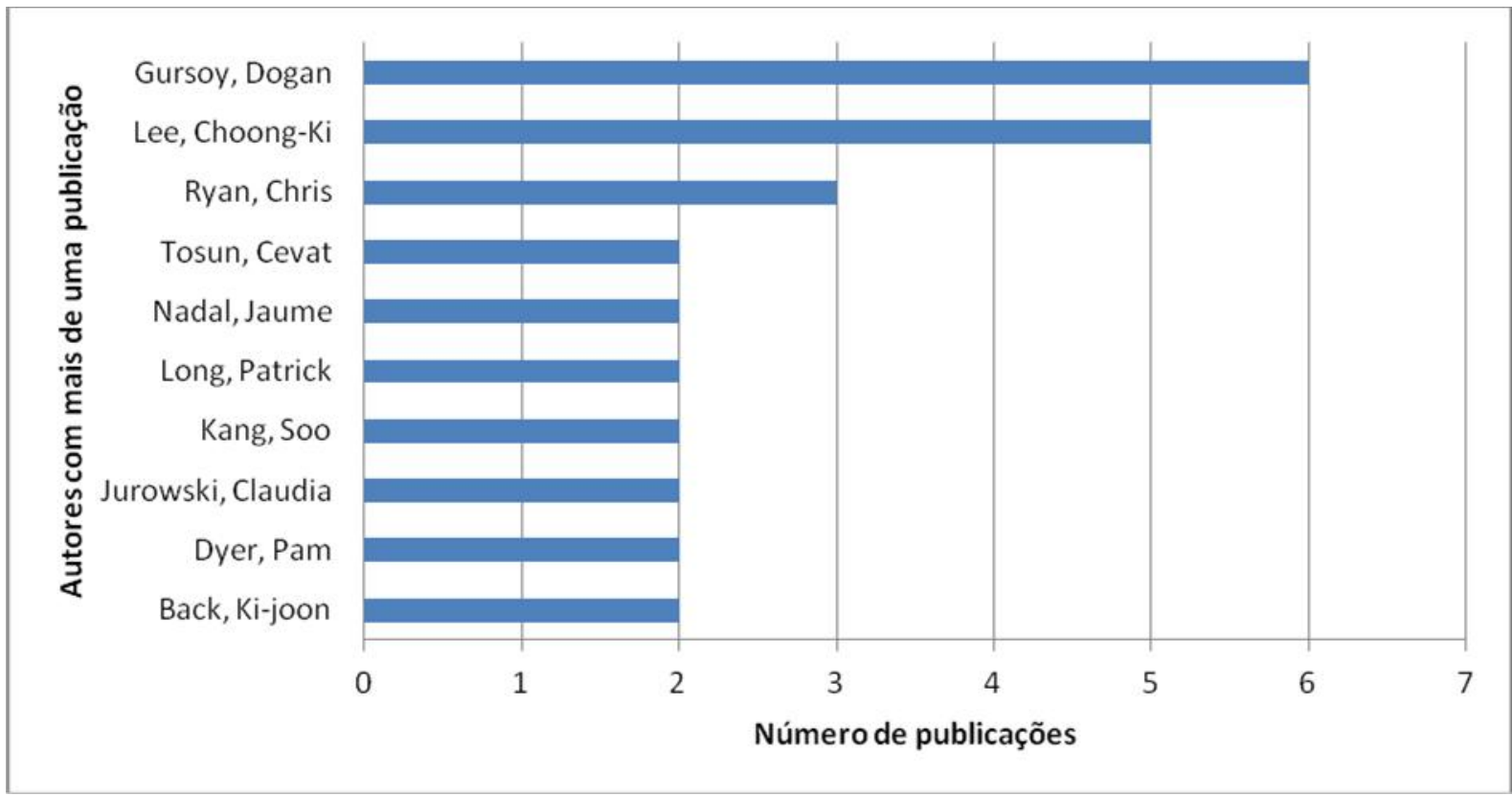

Gráfico 3: Autores com mais de uma publicação no tema

Fonte: Dados da pesquisa, 2012.

O fato de que existem autores que fazem 5 publicações ou mais em uma década, nas duas revistas internacionais mais relevantes da área e sobre o tema em questão, pode ser considerado um indicativo que existem especialistas internacionais sobre o tema. Esse é o caso de Gursoy (Estados Unidos) e Lee (Coreia do Sul), provenientes dos países com maior quantidade de publicações na referida abordagem.

Apesar do gráfico 3 apontar que existe um grupo de autores que estão focados na temática, é válido contrapor com o ranking dos principais autores na área de turismo (JOGARATNAMA, G., CHONB, K., McClEARYC, K. e MENAB, M., YOOB, J., 2005), para dimensionar até que ponto essa temática está sendo estudada por autores influentes do setor. Sendo assim, se identificou através de Jogaratnama et al, (2005) que Ryan, C. e Lee, Choong-Ki estão entre os 15 autores mais influentes dos periódicos de turismo, contraposto com os dados do gráfico 3, observa-se que esses estão entre os três pesquisadores mais presentes. Fato esse que sedimenta a importância do referido tema na área do turismo. 
A partir dos dados até o momento apresentados, ao considerar algumas correlações nesses contrastes, pode-se delimitar caminhos em prol de um aprimoramento das pesquisas no setor para o continente latino, mas que não pretendem esgotar a discussões sobre o tema e sim, iniciar as discussões sobre o tema. A grande maioria das publicações sobre a referida ótica nas revistas internacionais está focada em países desenvolvidos e de certa estabilidade social, entender como está o apoio da comunidade frente ao turismo em regiões em desenvolvimento como América Latina e África se mostram pertinentes e ainda pouco abordado.

As revistas analisadas no continente latino-americano possuem pesquisas sobre o referido assunto, o que mostra um interesse científico sobre o tema, mas a metodologia destoante com as referências no tema em âmbito internacional.

A metodologia SET, que foi encontrada na maioria absoluta das publicações internacionais, está presente em apenas $1(\mathrm{um})$ dos 15 (quinze) periódicos identificados no continente, na revista "Turismo em Análise", apesar da edição da revista ser brasileira, esse estudo foi feito por autores e sobre uma região de Portugal (GUERREIRO, M. M. ; MENDES, J. C. ; SILVA, J. A. , 2008), que, coincidentemente, é o único trabalho proveniente de fora do continente, ou seja, o único artigo que utiliza a metodologia SET é o único artigo proveniente de fora do continente latino, o que salienta o deslocamento do eixo estudado na temática, se comparado com as publicações internacionais.

Em um comparativo quanto à consolidação de autores especialistas nesse campo de estudo do turismo, nota-se que as revistas internacionais estão com autores de relevância trabalhando de forma constante sobre a temática, mas nas revistas latinas ainda estão carentes de profissionais da ciência que possam estudar e se aprofundar nesse assunto, possibilidade essa que forma uma sinergia com a necessidade de mais estudos dentro do marco teórico no continente.

Assim, um país como o Brasil, que irá receber nos próximos anos dois dos maiores megaeventos da atualidade, que possuem uma solidez social questionável e que não foi identificado na presente pesquisa nenhum estudo que utiliza a metodologia SET, poderia fazer uso desse marco teórico para aplicar estudos nesse eixo. Estudos de Gursoy (2006), autor especialista no tema com 6 publicações nos últimos 10 anos nas revistas internacionais 
selecionadas, tem em um dos seus estudos o título "Hosting megaevents: Modeling Locals' Support", que aponta um modelo teórico para analisar o apoio dos residente aos megaeventos:

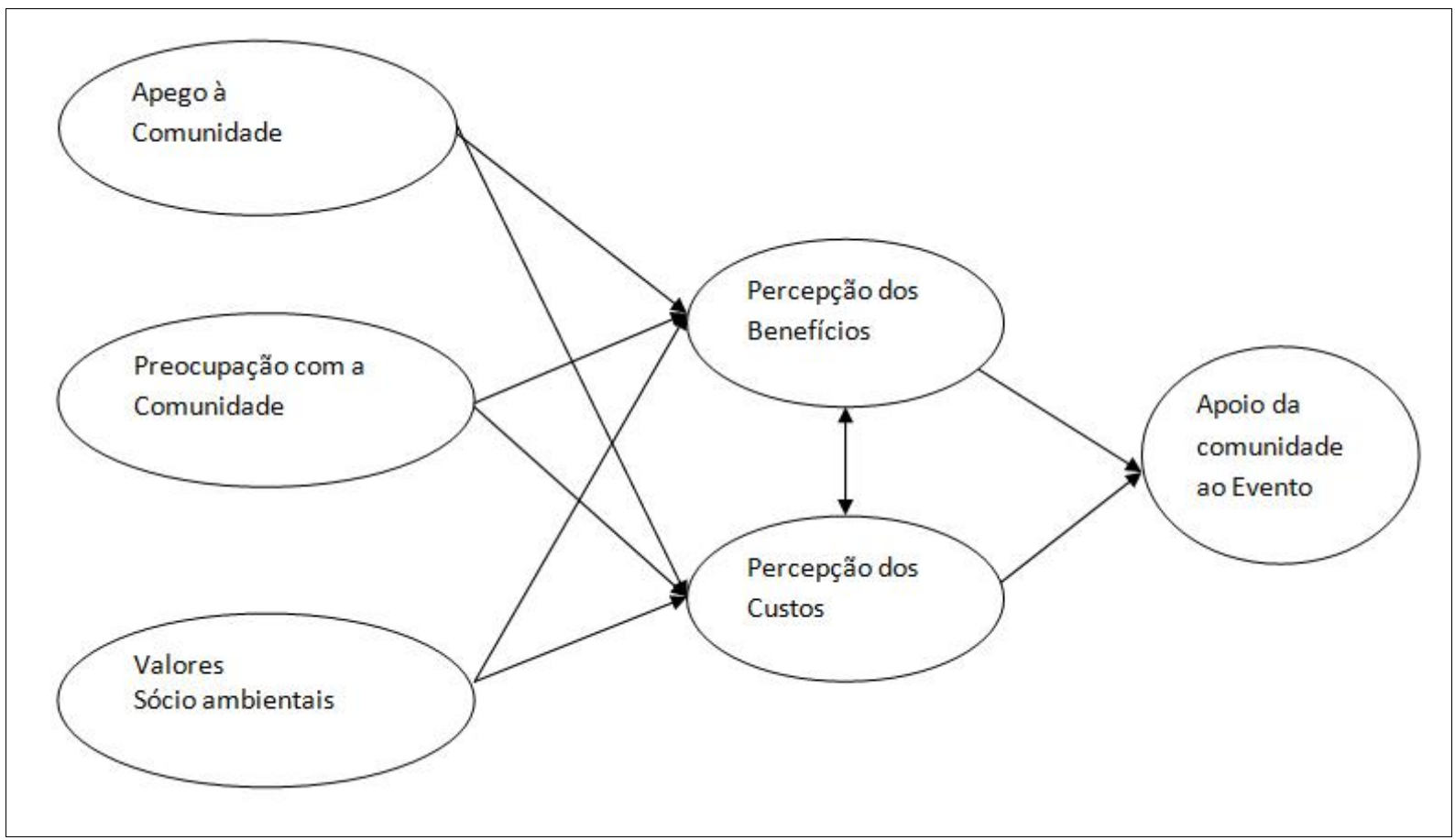

Figura1: Modelo de análise proposto por Gursoy (2006) para megaeventos.

Fonte: Adaptado de Gursoy, 2006.

Trabalhar sobre o marco teórico proposto não significa seguir de maneira inconteste as referências internacionais usadas para esse tipo de estudo, mas poder trabalhar sobre elas para poder aprimorá-las, falseá-las ou até validá-las no contexto latino- americano.

\section{Considerações Finais}

O presente estudo mapeou as pesquisas acadêmicas que versam sobre o olhar do residente sobre o turismo em âmbito mundial e latino-americano. $\mathrm{O}$ paralelo traçado entre esses dois contextos em estudo apresentou contrastes que podem ser úteis para o aprimoramento de pesquisas que versem sobre a referida temática. 
A partir do recorte proposto foi evidenciado que esse é um tema presente nas discussões acadêmicas, mas as publicações provenientes e voltadas para o continente latino estão à margem do marco teórico usado como referência no tema em âmbito internacional, contraste esse ainda mais acentuado se considerado o contexto vivido pelo continente em foco. Um dos fatores que pode explicar essa situação é a ausência de acadêmicos especializados nesse tipo de pesquisa na região.

Como parte dos objetivos propostos, foram sugeridos caminhos a serem adotados pelas futuras investigações sobre o tema no continente latino, a partir das referências internacionais, como um maior contingente de publicações sobre essa temática, uma adoção de pesquisadores que pretendem trabalhar especificamente no assunto e uso das técnicas e teorias como a SET, dentro dos contextos locais, como os megaeventos.

A presente investigação, apesar de não ter objetivado a questão generalista sobre os contrastes nas formas de pesquisar no continente latino-americano, apontou de forma indutiva, algumas diferenciações nas metodologias usadas, como uma predileção por técnicas qualitativas, o uso de teorias alternativas ao marco teórico vigente e uma barreira linguística marcante quanto a publicações entre os países de língua hispânica e lusa. Para validação dessas hipóteses faz-se necessário outros estudos que versem exclusivamente sobre essa temática.

Os resultados do presente estudo sugerem a necessidade de um aprimoramento das pesquisas nessa linha no continente latino-americano, além de outros estudos bibliométricos nessa mesma vertente com outros recortes da pesquisa, novas fontes de referências e outras categorias de análise como instituições, referências, pontuação de relevância de cada artigo e, principalmente, proposições de modelos teóricos vigentes na academia.

\section{Referências}

ALEGRE, J. e GARAU, J. Tourist satisfaction and dissatisfaction. Annals of Tourism Research, Vol. 37, n. 1, p. 52-73, 2010.

BID - Banco Interamericano de Desenvolvimento. http://www.iadb.org/pt/projetos/buscaavancada, 1301.html?adv=true . Acesso em 02/04/2012

BORSCHIEVER, S.; GUEDES, V. L. S. Bibliometria: Uma ferramenta estatística para a gestão da informação e do conhecimento, em sistemas de informação, de comunicação e de avaliação científica e tecnológica. Encontro Nacional da Ciência da Informação, Anais do CINFORM VI, Salvador, 2005. 
BRANDÃO, Carlos. A. O Processo de Subdesenvolvimento, as Desigualdades Espaciais e o jogo das escalas. In: Superintendência de Estudos Econômicos e Sociais. (Org.). Desigualdades Regionais. Salvador: SEI, p. 9-37, 2004.

$\begin{array}{llllll}\text { BRASIL. } & \text { Turismo no } & \text { Brasil } & & \text { 2011/ }\end{array}$ http://www.turismo.gov.br/export/sites/default/turismo/o_ministerio/publicacoes/downloads_publicac oes/Documento Referencial Turismo no Brasil 2011-2014.pdf. Acesso em 11/11/2011.

. Estatísticas básicas de turismo. Ministério do Turismo. Brasília. 2010

CAPES - Coordenação de Aperfeiçoamento de Pessoal de Nível Superior. Web-Qualis. Avaliação de Periódicos. Disponível em: <http://qualis.capes.gov.br/webqualis/>. Acesso em 01/12/2011.

CEBR - Centre for Economics and Business Research. <http://www.cebr.com/wpcontent/uploads/Cebr-World-Economic-League-Table-press-release-26-December-2011.pdf>. Acesso em 04/04/2012.

CURRIE, R. R., SEATON, S. e WESLEY, F. Determining stakeholders for feasibility analysis. Annals of Tourism Research, Vol. 36, No. 1, p. 41-63, 2009.

DREDGE, D. Place change and tourism development conflict: Evaluating public interest. Tourism Management, Vol.31, p. 104-112, 2010.

FONSECA, E. N. (organização); OTLET, P.; ESTIVALS R; ZOLTOWSKI. V.; GARFIELD. E. Bibliometria: Teoria e Prática. São Paulo: Cutrix. 1986.

JOGARATNAMA, G., CHONB, K., McCLEARYC, K. e MENAB, M., YOOB, J. An analysis of institutional contributors to three major academic tourism journals: 1992-2001. Tourism Management, Vol.26, p. 641-648, 2005.

GUERREIRO, M. M. ; MENDES, J. C. ; SILVA, J. A. Análise da satisfação dos residentes com o turismo: o caso de uma área-destino no Algarve, Portugal. Revista Turismo em Análise, São Paulo, dezembro, 2008.

GALEANO, E. As Veias Abertas da América Latina. Rio de Janeiro: Paz e Terra.1982

GIL, A. C. Métodos e técnicas de pesquisa social. 5. ed. São Paulo: Atlas, p.22 - 1994.

KRIPPENDORF, J. Sociologia do turismo: para uma nova compreensão do lazer e das viagens ( $3^{\mathrm{a}}$ ed.). São Paulo : Aleph. 2003.

GURSOY, D. , KENDALL, K.W. Hosting mega events: modelling locals' support.

Annals of Tourism Research, 33, 3: 603-623. 2006.

HALL, M. Publish and perish? Bibliometric analysis, journal ranking and the assessment of research quality in tourism. Tourism Management. V.32. p. $16-27,2011$.

MARREIRO, C. M. e MARQUES, S. Análise da relação causal entre imagem de destinos, qualidade, satisfação e fidelidade: Um estudo de acordo com a percepção do turista nacional no destino turístico Natal. Revista Turismo Visão e Ação - Eletrônica, Vol. 13 - nº 2 - p. 274-275 / mai-ago, 2011.

MENDONZA, M. M. O. ; TORRS, S. E. L. Turismo en Playa del Carmen - México Impactos socioculturales en la Colonia Colosio. Estudios y perspectivas en turismo. vol.19, no.5, set./out, 2010.

MONTERRUBIO, J. C. C.; MENDONZA, M. M. O.; LUQUE, A. G. Impactos sociales de la feria internacional del caballo en méxico: Una perspectiva local. Estudios y perspectivas en turismo. Vol.19, n.6, pp. 1090-1104, 2010. 
OMT - Organização Mundial do Turismo. http://unwto.org/en/content/why-tourism. Extraído dia $12 / 12 / 2011$.

PALOMO, M. F. Introducción al estudio económico del turismo. Barcelona: Civitas. 2000.

PANOSSO, N. A. Filosofia do Turismo: teoria e epistemologia. São Paulo: Aleph. 2005.

REJOWSKI, M. Produção científica em Turismo: análise de estudos referenciais no exterior e no Brasil. Revista Turismo e Análise. vol.21 n. 2, Agosto. 2010.

REJOWSKI, M.; KOBASHI, N. Y. Subsídios para Elaboração de um Tesauro Brasileiro de Turismo. Revista Turismo e Análise. Vol. 22, n. 3, dezembro. 2011.

RYAN, C. The ranking and rating of academics and journals in tourism research. Tourism Management, V.26, 657- 662, May. 2005.

SILVA, M. R. Análise bibliométrica da produção científica docente do programa de pós-graduação em educação especial da UFSCAR: 1998 - 2003. São Carlos - UFSCAR. Dissertação de Mestrado. 2005.

World Bank.http://data.worldbank.org/ acesso em 10/04/2012

Recebido em: 19/06/2012(1 ${ }^{\mathrm{a}}$ versão) $18 / 05 / 2013$ ( $2^{\mathrm{a}}$ versão)

Aprovado em: 25/06/2013 